\title{
Correction to: Fluorescence recovery after photobleaching: analyses of cyanobacterial phycobilisomes reveal intrinsic fluorescence recovery
}

\author{
Nan Zhang ${ }^{1,4} \cdot$ Kang Li $^{1,3} \cdot$ Bin-Bin Xie $^{1} \cdot$ Xiu-Lan Chen ${ }^{1} \cdot$ Bai-Cheng Zhou ${ }^{1} \cdot$ Hai-Nan Su ${ }^{1}$ Yu-Zhong Zhang ${ }^{1,2,3}$ \\ Published online: 25 June 2021 \\ (c) Ocean University of China 2021
}

\section{Correction to: Marine Life Science \& Technology https://doi.org/10.1007/s42995-021-00104-z}

The original version of this article unfortunately contained a mistake. A funding number was incorrect in the Acknowledgements section. The correct funding number is: Natural Science Foundation of Shandong (no. ZR2017LD013). The original article has been corrected.

The original article can be found online at https://doi.org/10.1007/ s42995-021-00104-z.

Hai-Nan Su

suhn@sdu.edu.cn

$\triangle$ Yu-Zhong Zhang

zhangyz@sdu.edu.cn

1 State Key Laboratory of Microbial Technology, and Marine Biotechnology Research Center, Shandong University, Qingdao 266237, China

2 College of Marine Life Sciences, Frontiers Science Center for Deep Ocean Multispheres and Earth System, Ocean University of China, Qingdao 266003, China

3 Laboratory for Marine Biology and Biotechnology, Pilot National Laboratory for Marine Science and Technology, Qingdao 266237, China

4 College of Bioengineering, Qilu University of Technology (Shandong Academy of Sciences), Jinan 250353, China 